> L'une des principales limitations de l'utilisation initiale des anticorps monoclonaux d'origine murine en thérapeutique était leur immunogénicité, entraînant leur neutralisation. Depuis, de nombreuses voies de recherche ont tenté avec succès de rapprocher les immunoglobulines (Ig) murines des anticorps humains et d'en améliorer ainsi l'efficacité et la tolérance chez l'homme. Deux grandes voies méthodologiques peuvent être utilisées : la biotechnologie, modifiant in vitro la séquence d'un anticorps animal, ou l'immunisation d'animaux dont le répertoire des gènes d'immunoglobulines a été préalablement humanisé. Dans ce dernier cas (objet de cette revue), des approches diverses ont permis de créer des souris transgéniques pour un nombre plus ou moins élevé de segments $V$ humains permettant d'obtenir un répertoire diversifié d'anticorps complètement humains. D'autres stratégies aboutissent à des $\mathrm{Acm}$ chimériques, le répertoire murin des segments VDJ réarrangés étant conservé et ceuxci s'associant à une région constante de chaîne lourde humaine. Les souris transgéniques humanisées constituent aujourd'hui des outils directs et séduisants, ayant permis le développement d'un nombre important d'anticorps de forte affinité et de faible immunogénicité dont certains sont en phase d'essai clinique et d'autres déjà commercialisés. <

\section{Transgenèse animale et humanisation des anticorps}

\section{Des souris}

pour des hommes

Michel Cogné, Sophie Duchez, Virginie Pascal

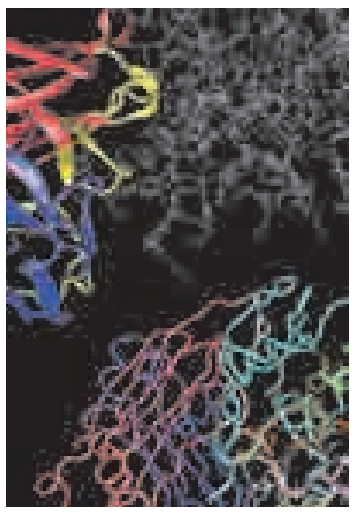

Université de Limoges, Laboratoire d'immunologie, Faculté de médecine, F-87025 Limoges, France; CNRS, UMR6101, Limoges, France. michel.cogne@unilim.fr

immunosuppresseur avant greffe d'organe $(\rightarrow)$. Immunogènes chez l'homme, ces anticorps animaux peuvent être neutralisés et/ou induire des $(\rightarrow)$ voir B. Vanhove, page 1121 réactions allergiques. Diverses voies ont donc été développées pour rapprocher les immunoglobulines (Ig) murines des anticorps humains et en améliorer l'efficacité et la tolérance chez l'homme. Cette «humanisation » peut être réalisée en retouchant in vitro la séquence d'un anticorps animal produit par biotechnologie $(\rightarrow)$, ou bien directement via l'immunisation

$(\rightarrow)$ voir A. Beck et al., d'animaux dont le répertoire page 1024 des gènes d'immunoglobulines a été préalablement humanisé. De nombreux anticorps issus de tels animaux sont en phase d'essai clinique ou déjà commercialisés et apparaissent comme des molécules particulièrement sûres. L'intérêt de ces animaux est lié au large répertoire d'antigènes qu'ils peuvent reconnaître, y compris quand il s'agit d'antigènes humains. S'y ajoute la possibilité de dériver ainsi tant des anticorps monoclonaux que polyclonaux (ou des associations oligoclonales). À l'inverse, le développement des méthodes de production d'anticorps humains à partir de lymphocytes humains reste limité en termes de répertoire, notamment vis-à-vis des auto-antigènes. 


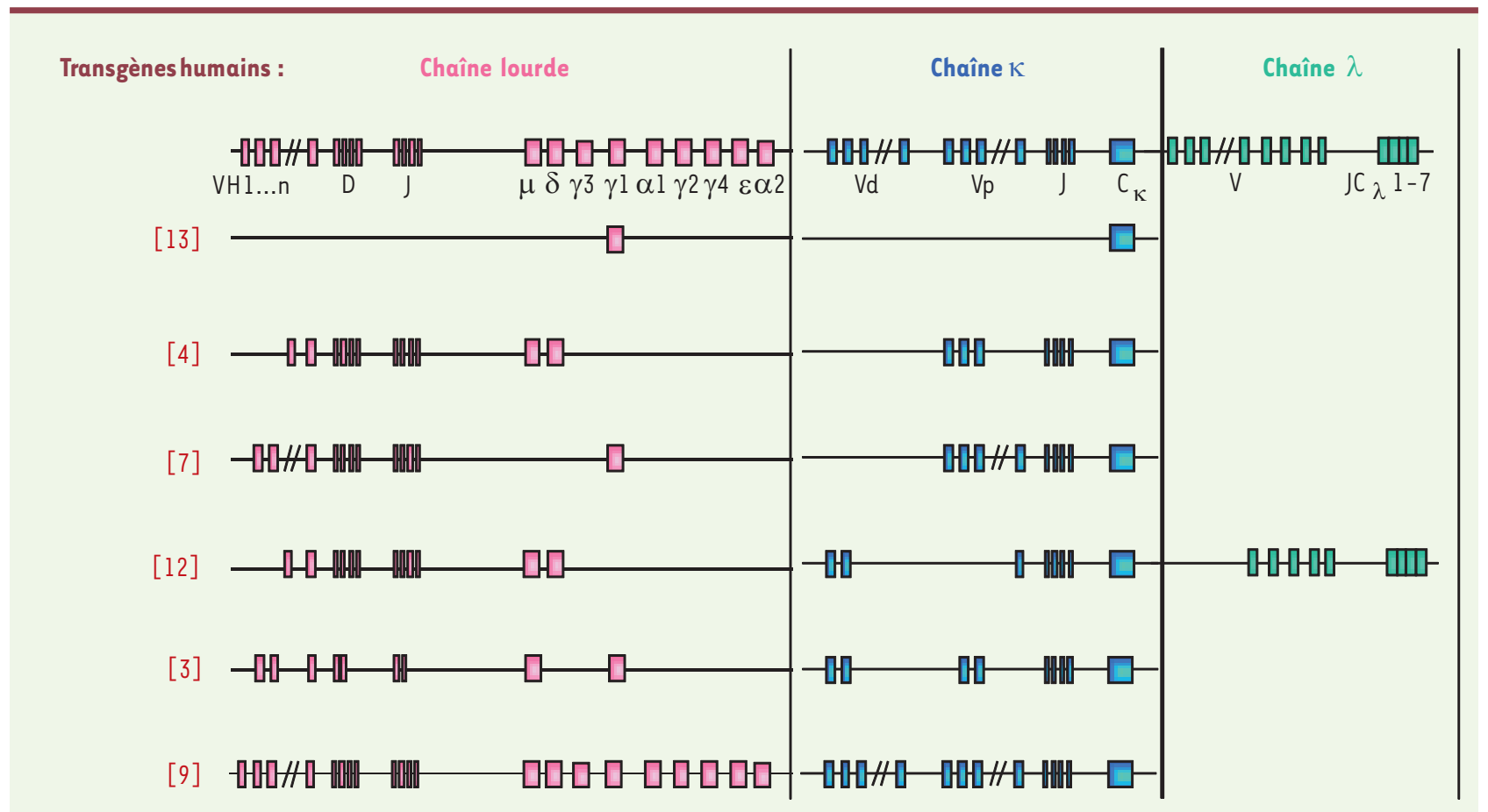

Figure 1. Transgènes d'immunoglobulines humaines introduits chez la souris. Les locus des chaînes lourdes $\mu, \delta, \gamma, \alpha$ et légères $\lambda$, $\kappa$, etc. sont représentés en configuration germinale. Les gènes introduits par insertion aléatoire dans le génome des souris transgéniques sont schématisés en précisant le nombre de segments $V$ et de gènes constants inclus dans les transgènes.

\section{Répertoire diversifié d'anticorps humanisés et chimériques chez la souris}

Alors que la transgenèse a pu être utilisée chez de grands mammifères tels que la chèvre pour la production abondante d'un anticorps de spécificité définie, le seul animal permettant à ce jour de reconstituer un répertoire diversifié d'anticorps humanisés est la souris. La première description de souris exprimant un répertoire restreint de gènes d'Ig humains date de 1989 [2]. L'utilisation de longs transgènes humains qui incluaient les séquences codant pour deux à cinq régions variables [capables de réarrangements $V(D) J]$ et plusieurs gènes codant pour les régions constantes (capables de subir la commutation de classe) a ensuite été combinée à l'inactivation des gènes endogènes codant les immunoglobulines de souris [3-5]. Ces modèles ont permis d'obtenir des hybridomes producteurs d'anticorps humains spécifiques malgré un répertoire $V$ assez restreint: en effet, une part notable de la diversité des anticorps provient de la diversité jonctionnelle puis de la maturation d'affinité par hypermutation somatique, part plus importante que celle que crée la diversité combinatoire des différents segments germinaux. Un nombre restreint de segments $V$ induit cependant des lacunes du répertoire, en particulier contre des antigènes polysaccharides connus comme ciblés par des anticorps aux séquences germinales sans besoin d'hypermutation somatique [6].
En restant dans des fonds génétiques murins déficients pour les gènes d'Ig endogènes, des lignées transgéniques disposant d'un nombre plus élevé de segments $V$ humains ont donc été générées. Ce qui améliore l'étendue du répertoire et permet la reconstitution de compartiments lymphocytaires B de taille plus proche de la normale [7, 8]. Ces lignées ont été produites tant par transgenèse classique que par fusion de cellules souches embryonnaires (ES) avec des protoplastes pour le transfert de chromosomes de levure porteurs de larges transgènes humains, ou encore par fusion de cellules ES avec des microcellules dérivées de fibroblastes humains et apportant un minichromosome humain. Des lignées de souris portant les locus IgH et Igא (HuMAb mouse de Medarex), ou portant en plus le locus $\lg \lambda$ (Xenomouse d'ABgenix) ont notamment ainsi été créées. Des anticorps de haute affinité ont été obtenus à partir de ces lignées. [9-12].

\section{Approches chimériques}

Des approches chimériques ont également été réalisées en parallèle: des lignées murines ont été établies chez lesquelles les réarrangements des segments $V$, D et J endogènes (murins) sont respectés, mais où les segments VDJ réarrangés peuvent s'exprimer en association à une région constante de chaîne lourde humaine 


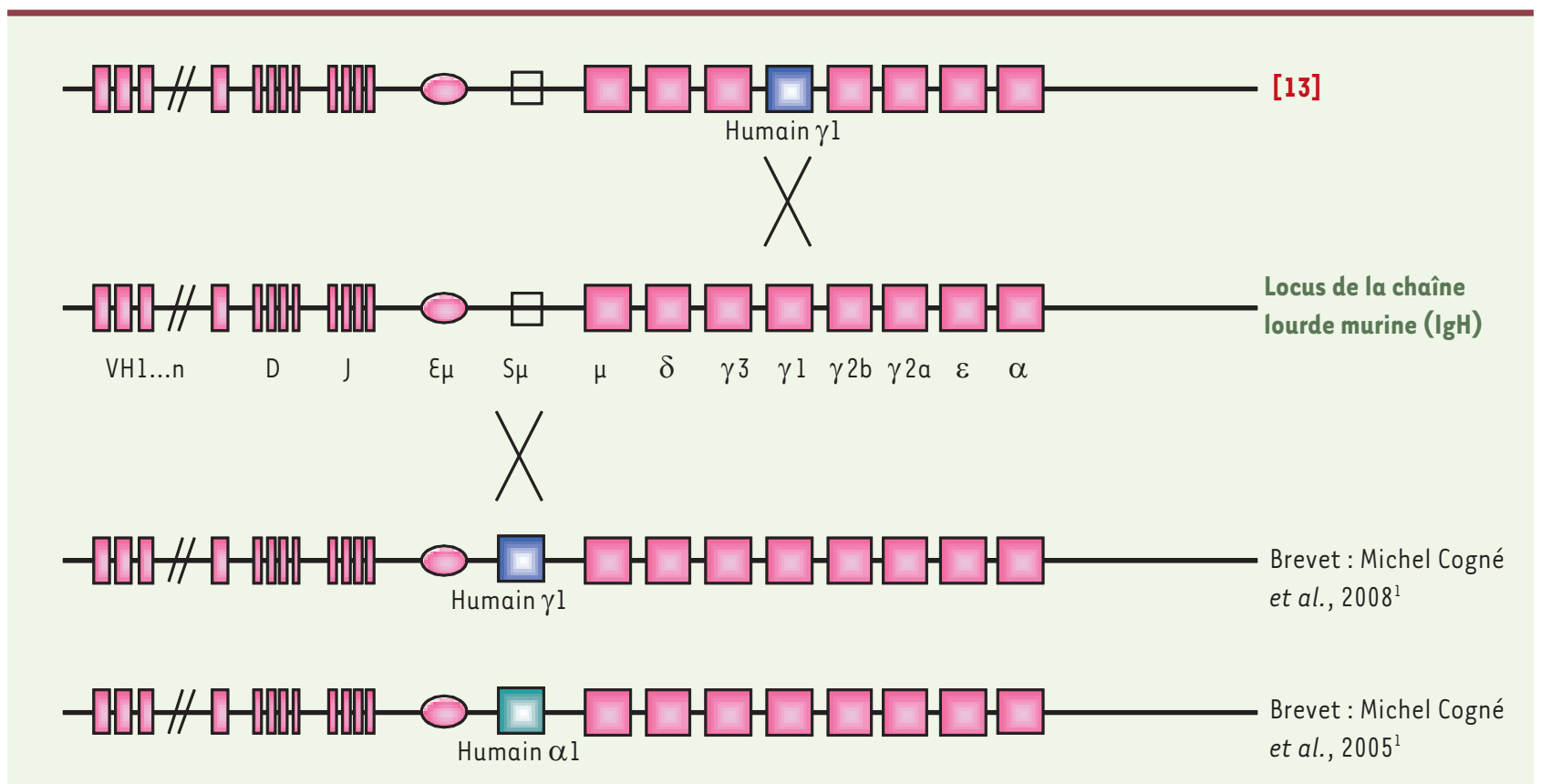

Figure 2. Séquences d'immunoglobulines humaines introduites dans le génome de la souris. Le locus des chaînes lourdes de la souris a été ciblé de façon à y introduire par recombinaison homologue des séquences provenant de régions constantes $\gamma$ ou $\alpha$ des gènes d'immunoglobulines humains.

(Figure 1). Ainsi, Zou et Rajewsky ont-ils remplacé les exons codant la partie sécrétée de la chaîne lourde $\gamma 1$ de l'IgGl murine par des exons $\gamma 1$ humains, substituant efficacement par des IgGl humaines chimériques les réponses humorales $\lg G 1$ de ces animaux, tout en gardant le répertoire VDJ murin [13]. Enfin, l'approche chimérique peut maintenant bénéficier de la disponibilité d'animaux qui correspondent simplement à l'insertion d'un gène codant pour la région constante humaine $\gamma l$ ou $\alpha \mathrm{l}$ en aval des clusters V, D et JH du locus IgH de la souris. Ces animaux permettent donc d'obtenir, d'emblée après fusion, des hybridomes spécifiques d'un antigène et dont l'isotype est prédéfini, soit $\operatorname{lgGl}$ soit IgAl, autorisant donc dès le stade des hybridomes initiaux les tests fonctionnels propres à l'isotype souhaité in fine (brevets Michel Cogné et al., 2005 ; Michel Cogné et al., 2008²) (Figure 2).

\section{Exemples d'anticorps thérapeutiques humanisés}

Tous ces animaux mutants sont caractérisés par une maturation lymphocytaire $B$ relativement normale en termes de taille des compartiments $B$, de réarrangements VDJ, de réarrangements de commutation de classe et d'hypermutation [14]. Ils ont ainsi permis de dériver un certain nombre d'anticorps de forte affinité et d'intérêt

${ }^{1}$ Cogné M, Sirac C, Bardel M, Decourt C, Laroche C, Brevet W02005047333, 2005, « Mammifère non humain transgénique pour la région constante de la chaîne lourde des IgA humaines et ses applications (monoclonaux humanisés à destinée muqueuse) 》.

Cogné $M$, Duchez $S$, Cogné $N$, Pinaud $\varepsilon$, Brevet 08/00319, 2008, « Mammifère non humain transgénique pour la région constante de la chaîne lourde des immunoglobulines humaines de classe $G$ et ses applications ». commercial important, reconnaissant aussi bien des petites molécules [15], des protéines spécifiques de pathogènes $[16,17]$, des polysaccharides [18], des protéines humaines solubles et des cytokines comme l'interleukine-15 [19-21] ou membranaires comme l'antigène spécifique de prostate PSMA, le CD20, le CD30 ou le récepteur à l'EGF (epidermal growth factor) [2224] et des antigènes associés à des tumeurs comme l'antigène carcinoembryonnaire [25].

Les premiers résultats à propos de tous ces Acm semblent très encourageants quant à leur faible immunogénicité chez l'homme $(\rightarrow)$. C'est le cas du panitumumab, anti-EGFR

$(\rightarrow)$ voir P. Stas et I. Lasters, page 1070 bloquant la liaison entre le ligand EGF et son récepteur (Abgenix/Amgen, Thousand Oaks, CA, USA). Les patients traités par le panitumumab ne développent pas d'anticorps polyclonaux contre l'anticorps théra-

$(\rightarrow)$ voir A. Beck et al., Tableau I, page 1026 peutique [26] $\rightarrow$ ).

Le denosumab est un autre anticorps apparemment très bien toléré [20] (Amgen, Thousand Oaks, CA, États-Unis). Il vise à combattre l'ostéoporose en ciblant le régulateur du remodelage osseux RANKL (receptor activator of $N K-K B$ ligand). La faible variabilité de la pharmacocinétique entre patients semble indiquer l'absence de réponse immune contre le desomumab. 
Même chez des patients porteurs de pathologies inflammatoires ou auto-immunes, la même absence de réponse immune a été observée visà-vis du zanolimumab [27] (Genmab, Copenhagen) qui lie le marqueur spécifique des lymphocytes T CD4 (avec un intérêt dans les lymphomes T et les pathologies auto-immunes). Même constat avec l'ipilimumab $[28,29]$ de Medarex (Princeton, NJ, USA) et Bristol-Myers Squibb (Wallingford, CT), qui en ciblant le récepteur inhibiteur CTLA-4 active les réponses immunes et est prescrit dans le mélanome. Globalement, les essais cliniques semblent donc indiquer une excellente tolérance de ces anticorps. Cette observation pourrait traduire une meilleure stabilité (des CDR3 et des paires $\mathrm{H}-\mathrm{L}$ ) des anticorps sélectionnés chez l'animal, alors corrélée à une moindre immunogénicité [30].

En revanche, d'autres $A c m$ thérapeutiques, qu'ils soient chimériques ou humanisés par greffe des CDR (complementary determining regions), voire issus de séquences humaines via des librairies de phage display, ont pu se révéler occasionnellement immunogènes en clinique [Humira anti-TNF (tumor necrosis factor), anti-IL-12, etc.] [31]. II convient d'ailleurs de noter que toute séquence $V$, même strictement humaine, peut être potentiellement immunogène chez l'homme et stimuler l'apparition d'antiidiotypes $(\rightarrow)$.

\section{$(\rightarrow)$ voir P. Stas et I. Lasters, page 1070}

\section{Une méthodologie d'avenir}

Outre le fait qu'elle permet l'obtention d'anticorps stables et peu immunogènes, l'utilisation d'animaux transgéniques présente l'intérêt de produire des molécules qui possèdent d'emblée les fonctions effectrices souhaitées pour la molécule thérapeutique finale, et peuvent donc entrer rapidement dans des tests de screening fonctionnel. À l'inverse, les régions variables créées artificiellement ou par phage display nécessitent un processus plus long de greffe sur des régions effectrices avant de pouvoir être validées et de permettre le choix des anticorps les plus intéressants dans une application et dirigés contre une cible donnée.

L'obtention d'hybridomes peut aussi permettre - en n'utilisant qu'un seul système d'expression - d'aller de la caractérisation initiale d'un anticorps jusqu'à sa production industrielle. Cependant, même s'agissant d'hybridomes, l'étape de la production industrielle à long terme substituera souvent aux cellules d'hybridomes l'utilisation de transfectants stables via des vecteurs d'expression dans lesquels auront été clonées les séquences Ig d'intérêt $(\rightarrow)$.

$\rightarrow$ voir 0 . Cochet et M. Chartrain, page 1078 ; S. Olivier et M. Mehtali, page 1163 Pour des applications où il serait intéressant de mimer une réponse physiologique et de reconstituer l'équivalent d'un sérum polyclonal ou oligoclonal capable de neutraliser simultanément un grand nombre d'épitopes sur un antigène complexe, l'utilisation d'animaux transgéniques humanisés est également intéressante parce qu'elle permet d'obtenir rapidement une variété d'anticorps de mêmes fonctions effectrices et possédant des paratopes et des affinités variés.

Pour l'ensemble de ces raisons, dans l'arsenal des stratégies d'obtention d'anticorps d'intérêt thérapeutique, les souris transgéniques humanisées constituent aujourd'hui des outils directs et séduisants. $\diamond$

\section{SUMMARY}

Transgenesis and humanization of murine antibodies The properties of monoclonal antibodies explain why they are such a successful class of therapeutic molecules. However, pionneered initial antibodies were of murine origin and triggered an immune response which limited the therapeutic potential of the antibody and generated deleterious effects. Consequently, tremendous efforts have been developped to engineer these murine Ig by introducing human sequences in vitro, or in vivo by humanization of murine antibodies, leading to chimeric immunoglobulins, and more recently generation of fully human antibodies in transgenic mice with a more or less diversified $V$ repertoire. These approaches have led to the development of an increasing number of these chimeric or humanized monoclonal antibodies entering pharmaceutical pipelines. $\diamond$

\section{CONFLIT D'INTÉRÊTS}

Les auteurs déclarent n'avoir aucun conflit d'intérêts concernant les données publiées dans cet article.

\section{RÉFÉRENCES}

1. Kohler G, Milstein C. Continuous cultures of fused cells secreting antibody of predefined specificity. Nature $1975 ; 256: 495-7$.

2. Bruggemann M, Caskey HM, Teale C, et al. A repertoire of monoclonal antibodies with human heavy chains from transgenic mice. Proc Natl Acad Sci USA 1989; $86: 6709-13$.

3. Lonberg N, Taylor LD, Harding FA, et al. Antigen-specific human antibodies from mice comprising four distinct genetic modifications. Nature $1994 ; 368: 856-9$.

4. Green LL, Hardy MC, Maynard-Currie CE, et al. Antigen-specific human monoclonal antibodies from mice engineered with human Ig heavy and light chain YAC. Nat Genet $1994 ; 7$ : 13-21.

5. Taylor LD, Carmack CE, Huszar D, et al. Human immunoglobulin transgenes undergo rearrangement, somatic mutation and class switching in mice that lack endogenous IgM. Int Immunol $1994 ; 6$ : 579-91.

6. Adderson $દ \varepsilon$, Shackelford PG, Insel RA, et al. Immunoglobulin light chain variable region gene sequences for human antibodies to Haemophilus influenzae type $b$ capsular polysaccharide are dominated by a limited number of V kappa and V lambda segments and VJ combinations. J Clin Invest $1992 ; 89: 729-38$.

7. Mendez MJ, Green LL, Corvalan JR, et al. Functional transplant of megabase human immunoglobulin loci recapitulates human antibody response in mice. Nat Genet 1997 ; $15: 146-56$.

8. Green LL, Jakobovits A. Regulation of B cell development by variable gene complexity in mice reconstituted with human immunoglobulin yeast artificial chromosomes. J Exp Med 1998 ; 188 : 483-95.

9. Tomizuka K, Shinohara T, Yoshida H, et al. Double trans-chromosomic mice: maintenance of two individual human chromosome fragments containing Ig heavy and kappa loci and expression of fully human antibodies. Proc Natl Acad Sci USA $2000 ; 97: 722-7$.

10. Kuroiwa Y, Tomizuka K, Shinohara T, et al. Manipulation of human minichromosomes to carry greater than megabase-sized chromosome inserts. Nat Biotechnol 2000 ; 18 : 1086-90.

11. Popov AV, Zou X, Xian J, Nicholson IC, Bruggemann M. A human immunoglobulin lambda locus is similarly well expressed in mice and humans. J Exp Med 1999; 189: 1611-20.

12. Nicholson IC, Zou X, Popov AV, et al. Antibody repertoires of four- and five-feature translocus mice carrying human immunoglobulin heavy chain and kappa and lambda light chain yeast artificial chromosomes. J Immunol 1999; 163: 6898-906. 


\section{RÉFÉRENCES}

13. Zou YR, Muller W, Gu H, Rajewsky K. Cre-loxP-mediated gene replacement: a mouse strain producing humanized antibodies. Curr Biol $1994 ; 4$ : 1099-103.

14. Harding FA, Lonberg N. Class switching in human immunoglobulin transgenic mice. Ann NY Acad Sci $1995 ; 764: 536-46$.

15. Ball WJ, Jr., Kasturi R, Dey P, et al. Isolation and characterization of human monoclonal antibodies to digoxin. J Immunol 1999; 163: 2291-8.

16. Mukherjee J, Chios K, Fishwild D, et al. Human Stx2-specific monoclonal antibodies prevent systemic complications of Escherichia coli 0157 : H7 infection. Infect Immun 2002 ; $70: 612-9$.

17. He Y, Honnen WJ, Krachmarov CP, et al. Efficient isolation of novel human monoclonal antibodies with neutralizing activity against HIV-l from transgenic mice expressing human Ig loci. J Immunol 2002; 169:595-605.

18. Chang $Q$, Zhong $Z$, Lees $A$, et al. Structure-function relationships for human antibodies to pneumococcal capsular polysaccharide from transgenic mice with human immunoglobulin Loci. Infect Immun 2002; 70 : 4977-86.

19. Villadsen LS, Schuurman J, Beurskens F, et al. Resolution of psoriasis upon blockade of IL-15 biological activity in a xenograft mouse model. J Clin Invest $2003 ; 112$ : 1571-80.

20. Bekker PJ, Holloway DL, Rasmussen AS, et al. A single-dose placebo-controlled study of AMG 162, a fully human monoclonal antibody to RANKL, in postmenopausal women. J Bone Miner Res $2004 ; 19: 1059-66$.

21. Yang XD, Corvalan JR, Wang $P$, et al. Fully human anti-interleukin- 8 monoclonal antibodies potential therapeutics for the treatment of inflammatory disease states. J Leukoc Biol 1999; 66: 401-10.

22. Teeling JL, French RR, Cragg MS, et al. Characterization of new human CD20 monoclonal antibodies with potent cytolytic activity against non-Hodgkin lymphomas. Blood 2004 ; $104: 1793-800$

23. Schuler W, Bigaud M, Brinkmann V, et al. Efficacy and safety of $A B 1793$, a novel human anti-human CD154 monoclonal antibody, in cynomolgus monkey renal allotransplantation. Transplantation $2004 ; 77: 717-26$.
24. Bleeker WK, Lammerts van Bueren JJ, van Ojik HH, et al. Dual mode of action of a human anti-epidermal growth factor receptor monoclonal antibody for cancer therapy. J Immunol $2004 ; 173$ : 4699-707.

25. Imakiire T, Kuroki M, Shibaguchi $\mathrm{H}$, et al. Generation, immunologic characterization and antitumor effects of human monoclonal antibodies for carcinoembryonic antigen. Int J Cancer $2004 ; 108: 564-70$.

26. Foon KA, Yang XD, Weiner LM, et al. Preclinical and clinical evaluations of ABX-EGF, a fully human anti-epidermal growth factor receptor antibody. Int J Radiat Oncol Biol Phys 2004 ; 58 : 984-90.

27. Skov L, Kragballe K, Zachariae C, et al. HuMax-CD4 : a fully human monoclonal anti-CD4 antibody for the treatment of psoriasis vulgaris. Arch Dermatol 2003 ; 139 : 1433-9.

28. Keler T, Halk $\varepsilon$, Vitale L, et al. Activity and safety of CTLA-4 blockade combined with vaccines in cynomolgus macaques. J Immunol 2003; $171: 6251-9$

29. Phan GQ, Yang JC, Sherry RM, et al. Cancer regression and autoimmunity induced by cytotoxic T lymphocyte-associated antigen 4 blockade in patients with metastatic melanoma. Proc Natl Acad Sci USA 2003; $100: 8372-7$.

30. Hermeling S, Crommelin DJ, Schellekens H, Jiskoot W. Structureimmunogenicity relationships of therapeutic proteins. Pharm Res 2004 ; $21: 897-903$

31. Mannon PJ, Fuss IJ, Mayer L, et al. Anti-interleukin-12 antibody for active Crohn's disease. N Engl J Med 2004 ; 351 : 2069-79.

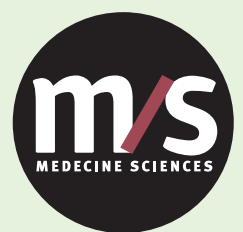

Tarifs d'abonnement M/S - 2010

Abonnez-vous

à Médecine/Sciences
> Depuis 20 ans, grâce à $m / s$, vous vivez en direct les progrès des sciences biologiques et médicales

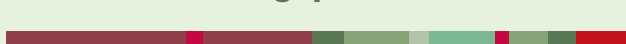

$$
\begin{gathered}
\text { Bulletin d'abonnement } \\
\text { page VI dans ce numéro de } \mathrm{m} / \mathrm{s}
\end{gathered}
$$




\section{Hépatite C}

Jean-Michel Pawlotsky Daniel Dhumeaux

e virus de l'hépatite C a été identifié par l'équipe de Michael Houghton en 1989.

De nombreux progrès ont été accomplis. Une proportion importante des 500000 malades français a été identifiée. Beaucoup de ces malades ont pu être pris en charge, traités et, pour une part non négligeable, guéris. Un ouvrage de référence surl'hépatite Cécrit en français par des spécialistes francophones de renommée internationale.

Ce livre fait le point des connaissances, alors que les traitements actuels semblent avoir atteint le maximum de ce qu'ils pouvaient offrir et que de nouvelles approches thérapeutiques voient le jour.

Jean-Michel Pawlotsky

Daniel Dhumeaux

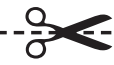

À retourner à EDK, 2, rue Troyon - 92310 Sèvres

Tél. : 0155641393 - Fax : 0155641394 - ع-mail : edk@edk.fr

NOM : Prénom :

Adresse :

Code postal : Ville :

Pays :

Fonction :

Je souhaite recevoir l'ouvrage

Hépatite $\mathrm{C}: 60 €+3 €$ de port $=63 €$ TTC

en ................. exemplaire, soit un total de $€$

$\square$ Par chèque, à l'ordre de EDK

$\square$ Par carte bancaire : Visa Eurocard/Mastercard

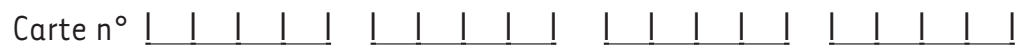
Signature :

$\mathrm{N}^{\circ}$ de contrôle au dos de la carte :

Date d'expiration : 